\title{
PROMOÇÃO DA SAÚdE PARA PREVENÇÃO E ENFRENTAMENTO DO ALZHEIMER, PARKINSON E AVE
}

\author{
HEALTH PROMOTION FOR PREVENTION AND COPING OF \\ ALZHEIMER, PARKINSON AND AVE
}

\author{
Karla Pereira Vasconcelos ${ }^{1}$ \\ Cleisla Thamires Lacerda Silva ${ }^{2}$ \\ Hirisdiane Bezerra Alves ${ }^{3}$
}

RESUMO: OBJETIVO: Promover a orientação da terceira idade acerca do Alzheimer, Parkinson e AVE, a fim de contribuir para o conhecimento das temáticas, com ênfase na prevenção e formar multiplicadores do conhecimento. METODOLOGIA: Trata-se de um relato de experiência de estágio extracurricular, realizado na empresa Sesc (Unidade Açude Velho) em Campina-Grande na Paraíba. As ações foram realizadas no período de agosto a dezembro de 2019. Os temas abordados foram voltados à promoção da saúde para prevenção e enfrentamento do Alzheimer, Parkinson e AVE, com ênfase na prevenção dos distúrbios. As práticas educativas utilizadas para abordar as temáticas foram constituídas de dinâmicas, palestras e roda de conversa. RESULTADOS: Os índices de acometimento de idosos pelo Alzheimer, Parkinson e AVE são altos, e apesar disso, ainda há pouca discursão acerca das temáticas, sendo possível observar que a terceira idade tem pouco ou nenhum conhecimento sobre os assuntos. Após a aplicação das atividades, houve melhoras significativas no entendimento do público-alvo acerca dos distúrbios citados, sendo possível perceber que ao final da aplicação do plano, os idosos souberam responder perguntas, falar sobre os temas e compartilharam experiências positivas sobre os encontros. CONCLUSÃO: Com a aplicação do plano de estágio, foi possível perceber quer os conhecimentos sobre o Alzheimer, Parkinson e AVE são deficientes e poucos difundidos, e os índices de acometimento são elevados, sendo de extrema importância que as temáticas sejam abordadas para toda a população, e em especial a população idosa que tem maior incidência de acometimento por esses distúrbios.

\footnotetext{
1 Graduanda do Curso de Enfermagem da Universidade Estadual da Paraíba - UEPB, karlapv2017@gmail.com;

2 Graduanda do Curso de Enfermagem da Universidade Estadual da Paraíba - UEPB, karlapv2017@gmail.com;

${ }^{3}$ Enfermeira pelo Centro Universitário Maurício de Nassau/ Campina Grande. Pós-graduanda em Urgência, Emergência e UTI pelo Instituto Michele Sales/ Campina Grande. Pós-graduanda em Obstetrícia e Neonatologia pelo Instituto Michele Sales/ Campina Grande. dianyalves06@gmail.com.
} 
Palavras chave: Alzheimer. Idosos. Promoção da saúde.

ABSTRACT: OBJECTIVE: To promote the orientation of the elderly about Alzheimer's, Parkinson's and CVA, in order to contribute to the knowledge of the themes, with an emphasis on prevention and to form knowledge multipliers. METHODOLOGY: This is an experience report of an extracurricular internship, carried out at the company Sesc (Unidade Açude Velho) in Campina-Grande, Paraiba. The actions were carried out from August to December 2019. The topics covered were focused on health promotion for the prevention and coping of Alzheimer's, Parkinson's and CVA, with an emphasis on the prevention of disorders. The educational practices used to address the themes were made up of dynamics, lectures and conversation. RESULTS: The rates of involvement of the elderly by Alzheimer's, Parkinson's and CVA are high, and despite this, there is still little discussion about the themes, being possible to observe that the elderly have little or no knowledge about the subjects. After the application of the activities, there were significant improvements in the understanding of the target audience about the aforementioned disorders, and it is possible to notice that at the end of the application of the plan, the elderly knew how to answer questions, talk about the themes and shared positive experiences about the meetings. CONCLUSION: With the application of the internship plan, it was possible to perceive that knowledge about Alzheimer's, Parkinson's and CVA are deficient and few disseminated, and the rates of involvement are high, being extremely important that the themes are addressed to the entire population, and in particular the elderly population that has a higher incidence of involvement by these disorders.

Keywords: Alzheimer's. Seniors. Health promotion. 


\section{INTRODUÇÃO}

O processo de envelhecimento da população leva a mudanças no cenário da saúde, caracterizado pelo aumento de doenças crônicas não transmissíveis, destacando- se a depressão e as demências (HELLWIG; MUNHOZ; TOMASI, 2016). Segundo a Organização Pan-Americana da Saúde, demência é um termo "guardachuva" utilizado para definir diversas doenças que são progressivas, afetam a memória, capacidades cognitivas (habilidades motoras, atenção e percepção) e interfere no desenvolvimento de atividades cotidianas (OPAS, 2017). No Brasil tem sido observado um aumento progressivo da incidência de doenças demenciais, sendo este associado ao envelhecimento da população, e a doença de Alzheimer é a mais prevalente dessas demências (SILVA; RIBEIRO; MARTINEZ, 2017).

A Doença de Alzheimer (DA) é uma afecção neurodegenerativa, crônica e progressiva, de aparecimento insidioso, que causa perda da memória associada a diversos distúrbios cognitivos, sendo mais comum na população acima de 60 anos (BRASIL, 2013). Estudos recentes demonstram que no Brasil, a taxa de prevalência de demência na população com mais dos 65 anos foi de $7,1 \%$, sendo a doença de Alzheimer responsável por $55 \%$ dos casos. A principal causa ainda é desconhecida, mas, acredita-se que pode estar associada a fatores genéticos. A doença pode ser dividida em quatro estágios (estágio 1, 2, 3 e 4), e a sobrevida média de pessoas com DA costuma oscilar entre 8 a 10 anos.

Indivíduos acometidos com DA, podem apresentar falta de memória para acontecimentos recentes, repetição de perguntas, dificuldade de acompanhar pensamentos, dificuldade para expressar palavras, encontrar caminhos conhecidos, dentre outros sintomas, que variam de acordo com o estágio. Os principais fatores de risco para o desenvolvimento do Alzheimer incluem: idade avançada, histórico familiar, sedentarismo, estilo de vida e alimentação inadequada (BRASIL, 2019).

Além da Doença de Alzheimer, o Parkinson e o Acidente Vascular Encefálico (AVE) constituem-se de afecções crônicas comumente encontradas em indivíduos 
idosos, sendo a Doença de Parkinson uma doença neurológica que possui como principais características os tremores e lentidão dos movimentos e se apresenta de modo comum e intrigante, visto que possui distribuição universal e pode atingir todas as classes sociaise etnias (BRASIL, 2014).

A Doença de Parkinson ocorre por causa da degeneração das células da substância negra. Essas células são responsáveis pela produção da dopamina, um importante neurotransmissor que conduz as correntes nervosas pelo corpo. Quando ocorre a diminuição ou falta desse neurotransmissor, o indivíduo tem seus movimentos afetados (BRASIL, 2012). Esse distúrbio atinge cerca de $1 \%$ da população mundial com idade superior a 65 anos, e no Brasil estima-se que cerca de 200 mil pessoas sofram com o problema (BRASIL, 2014). Apesar de não existir cura para a doença, existem tratamentos que visam reduzir os sintomas e retardar o seu progresso, contribuindo para melhorar a qualidade de vida dos acometidos.

O Acidente Vascular Encefálico constitui-se de um importante problema de saúde pública, com altos índices de sequelas e morte, além de se configurar pelo seu forte impacto social e econômico (SANTANA; CHUN, 2016), estando entre as 10 principais causas de morte entre as doenças crônicas não transmissíveis (DNCT), segundo dados de 2018 da Organização Pan-Americana de Saúde, ficando atrás apenas da cardiopatia isquêmica, sendo a cardiopatia isquêmica e o AVE os responsáveis por um total de 15,2 milhões de óbitos no ano de 2016 (OPAS, 2018).

O AVE possui dois tipos principais: hemorrágico e isquêmico. O hemorrágico ocorre devido ao rompimento do vaso, gerando uma hemorragia, que pode ocorrer tanto dentro do tecido cerebral quanto na superfície entre o cérebro e a meninge. Por outro lado, o isquêmico é causado pela obstrução do vaso, o que gera falta de oxigenação para células cerebrais, sendo o primeiro responsável por $15 \%$ dos casos de AVE, enquanto o segundo corresponde a maior parte dos casos, somando $85 \%$ (Brasil, 2018). Os principais sintomas do AVE incluem: fraqueza, confusão mental, formigamento, alterações visuais, dor de cabeça súbita, dentre outros. Os fatores de risco para o desenvolvimento da patologia podem ser divididos em duas categorias, os fatores modificáveis e os não modificáveis. Os não modificáveis são aqueles que não se pode intervir ou modificar, a exemplo da idade, sexo, raça/cor e genética/história familiar, já os modificáveis são aqueles nos quais podemos intervir 
e como consequência prevenir e tratar, sendo eles; hipertensão arterial (mais significativo), doenças cardiovasculares, dislipidemias, tabagismo, alcoolismo, sedentarismo, dentre outros (CARVALHO; DEODATO, 2016).

A Pesquisa Nacional de Saúde, no ano de 2013, revelou que cerca de 2,2 milhões de adultos haviam sofrido um Acidente Vascular Encefálico, sendo este, um importante problema socioeconômico devido a seus agravos e sequelas como: dificuldades nos domínios de funções de orientação, na função da memória, funções mentais de linguagem, dificuldades de concentração, escrita, caminhar, assim como, implicações na participação em seu meio social, recreação e lazer (SANTANA; CHUN, 2016).

Considerando que as doenças crônicas não transmissíveis constituem o problema de saúde pública de maior relevância, correspondendo a $72 \%$ das causas de mortes (BRASIL, 2011). A população idosa é a mais acometida por agravos crônicos não transmissíveis, comorbidades que tendem a comprometer a qualidade de vida, afetando principalmente a funcionalidade e desenvolvimento das atividades cotidianas (BRASIL, 2011). Dentre esses agravos podemos destacar: a Doença de Alzheimer, o Parkinson e o Acidente Vascular Encefálico. Assim, a educação em saúde possui papel relevante na conscientização da população, prevenção de agravos e sequelas e na formação de cidadãos conscientes do seu papel social, e multiplicadores do conhecimento, sendo de extrema importância que a população idosa, tenha conhecimento sobre as causas, fatores de risco, manifestações clínicas, prevenção e tratamento dessas patologias, visto que é o público de maior ocorrência dessas doenças, mas, possuem informações limitadas acerca dessas temáticas.

Desse modo, podemos afirmar que a educação em saúde se constitui de uma ferramenta primordial na transmissão de informações, devendo ser valorizada como instrumento fundamental na assistência, controle efetivo e prevenção das complicações crônicas (SALCl; MEIRELLES; SILVA, 2017) visando promover a saúde por meio de práticas educativas referente aos principais distúrbios neurológicos encontrados em idosos, visto que é de extrema importância por se tratar de um grupo que apresenta dificuldade de se relacionar por possuir maior tendência ao isolamento (NÓBREGA et al., 2015), que gera limitação no acesso as 
informações que são importantes para sua qualidade de vida e seu bem estar holístico.

\section{METODOLOGIA}

O presente artigo trata-se de um relato de experiência de estágio extracurricular realizado na empresa Sesc (Unidade Açude Velho, em Campina Grande/PB), no período agosto a dezembro de 2019, onde o plano desenvolvido abordou a promoção da saúde para prevenção e enfrentamento do Alzheimer, Parkinson e AVE, cujo público-alvo foi a terceira idade. As atividades foram supervisionadas pelas enfermeiras Audrey Sanny Alves de Freitas e Daysianne Monalise. O plano foi realizado com 80 idosos participantes do grupo "Balança, mas, não cai". As reuniões aconteciam nas quintas-feiras, no turno da manhã, das 9:00 as 9:45. O grupo era composto de 78 mulheres e 2 homens. As atividades desenvolvidas foram fundamentadas na realização de dinâmicas, palestras educativas e roda de conversa, a fim de que o grupo adquirisse o conhecimento suficiente sobre as temáticas abordadas. Cada encontro abordou um tema específico. O primeiro encontro foi destinado a realização de dinâmica, bem como, roda de conversa sobre Alzheimer, Parkinson e AVE, a fim de sondar o conhecimento do público-alvo sobre os temas para assim confeccionar o material para as palestras. Para a elaboração do material foi considerado também a escolaridade onde a maioria dos idosos tinham apenas ensino fundamental incompleto. A primeira palestra abordou o tema Alzheimer, a segunda foi sobre Parkinson e a última AVE. As atividades ocorreram de modo expositivo por meio de palestras educativas e tiveram como objetivo fazer com que os idosos participantes do grupo "Balança, mas não cai" adquirisse conhecimento suficiente sobre o que é, as principais causas, manifestações clínicas, prevenção e tratamento dos distúrbios neurológicos mais incidentes em idosos (Alzheimer, Parkinson e AVE). A cada encontro era abordado um dos temas de modo simples, claro e dinâmico. Para tanto eram utilizados slides como principal material demonstrativo, proporcionando a utilização de imagens para manter o 
conteúdo mais atrativo. Ao final da apresentação de cada temática, eram feitas perguntas para verificar a aprendizagens dos idosos, bem como era aberto espaço para possíveis questionamentos.

\section{RESULTADOS E DISCURSÃO}

A reunião inicial compreendeu a apresentação ao grupo das temáticas que seriam abordadas durante os encontros, bem como, foram feitas perguntas sobre os temas para verificar o conhecimento do grupo sobre os conteúdos propostos, para assim poder confeccionar o material que seria utilizado. O encontro contou ainda com uma dinâmica que objetivou testar a memória dos idosos. Para isso, foi utilizada uma caixa e dentro desta havia vários objetos (carretel de linha, relógio, pente, caneta, dentre outros) e um por um deveriam ser tocados e o idoso deveria falar em voz alta o nome do objeto que havia sido tocado, em seguida retirá-lo da caixa e mostrar para o grupo. Essa atividade é proposta para detectar possíveis sinais de Alzheimer, visto que o indivíduo com essa patologia passa a esquecer de objetos simples do dia-a-dia. A dinâmica foi muito bem aceita e todos se divertiram, bem como, todos foram capazes de adivinhar o objeto ao qual havia sido tocado. Ao término da dinâmica foi realizada uma roda de conversa, onde os idosos foram perguntados o que entendiam sobre o Alzheimer, Parkinson e o AVE, aos poucos eles foram falando sobre experiências com conhecidos, mas, não sabiam ao certo definir os distúrbios.

Após o encontro inicial, foi marcado um novo encontro para a quinta-feira seguinte. Nesse encontro a temática abordada foi a Doença de Alzheimer. $O$ encontro começou com algumas imagens sendo expostas via slide para ao término da palestra ser perguntado quais eram essas imagens e assim tornar o encontro mais dinâmico, como também, testar a memória dos participantes. Logo após foi levantado o questionamento sobre o que era o Alzheimer, e uma idosa respondeu: “ é uma doença onde a pessoa esquece as memórias recentes". Foi explicado então, que a doença apresenta vários graus de acometimento, onde a pessoa acometida 
em estágio inicial, apresenta sim essa característica, mas, à medida que a doença evolui o indivíduo acometido esquece também memórias passadas e com o agravamento do quadro, o indivíduo esquece como desenvolver tarefas simples, como por exemplo; andar, comer e engolir. Fora levantado ainda, um questionamento acerca do modo de prevenção e então foi explicado a importância de treinar a mente, ler, estudar, praticar atividade física, não fumar, não beber, comer frutas e verduras, ingerir alimentos ricos em vitamina B12, e manter hábitos de vida saudável.

Ao término do encontro, uma senhora nos abordou e agradeceu pela palestra, visto que o seu esposo havia sido diagnosticado com a DA, mas nenhum dos profissionais as quais eles foram, explicou de que se tratava e afirmou para os colegas que a palestra havia sido melhor que muitas consultas as quais ela foi.

O terceiro encontro, abordou a temática: Doença de Parkinson, na qual foi apresentado as seguintes questões: o que é o Parkinson, as causas, as manifestações clínicas, os estágios, a prevenção e o tratamento. Inicialmente foi feito a pergunta sobre o que eles entendiam a respeito da patologia e alguns dos idosos simplesmente falaram que conheciam pessoas com a doença, mas não sabia ao certo do que se tratava, sabia apenas que causava tremor nas mãos. Assim foi explicada a fisiopatologia da doença, onde foi mostrado a parte do cérebro que era atingido (diminuição da substância negra), foi relatado que a principal causa ainda é desconhecida e que infelizmente não existe modo de prevenção conhecido que seja eficaz contra a patologia, mas alguns estudos demostraram que os índices de acometimento eram menores em indivíduos que praticavam atividades físicas regulares. Foi falado ainda, sobre a importância de levar um estilo de vida saudável, evitar o estresse, visando a diminuição dos tremores, uma das idosas compartilhou conosco sua experiência com seu pai, que tinha vivencia com a patologia e confirmou que os resultados do tratamento com o fármaco vinham sendo satisfatórios. Ao final do encontro foi levantada a seguinte questão: "quando desconfiar que um tremor pode ser Parkinson ?", e os idosos diante o que foi exposto souberam responder que o tremor do Parkinson era tremor de repouso e que havia piora quando o indivíduo estava estressado. Ao término do encontro, um dos idosos nos procurou e afirmou que estava ansioso para a palestra de AVE. 
No último encontro foi debatido sobre o Acidente Vascular Encefálico (AVE). A princípio foi falado sobre a mudança da nomenclatura, onde a nova nomenclatura visa ampliar o conceito, pois pode estar envolvida qualquer estrutura encefálica e não apenas a parte cerebral. Dando prosseguimento, foi apresentado os dois principais tipos de AVE (isquêmico e hemorrágico), explicado a fisiopatologia dos dois tipos, onde o primeiro ocorre pela interrupção do fluxo de sangue para o cérebro gerado por uma obstrução, e o segundo ocorre de vida ao rompimento do vaso, enfatizando a importância da prevenção por meio do estilo de vida saudável, a exemplo do controle do colesterol e da pressão arterial, visto que o AVE isquêmico tem como principal causa a deposição de placas de gorduras no vaso (aterosclerose) e o hemorrágico, tem como principal causa a hipertensão arterial. Durante a palestra foi demostrado os sinais de alerta para o AVE, onde foi ensinado que em casos com suspeita de AVE deve-se aplicar uma escala de avaliação pré-hospitalar (Escala de Cincinnati). A escala tem por objetivo avaliar parâmetros como: assimetria facial, debilidade dos membros e a incapacidade da fala, desse modo, foi ensinado como aplicá-la em casos suspeitos do seguinte modo: pede-se para a pessoa sorrir e terá resposta inadequada o indivíduo que apresentar assimetria facial; deve-se pedir para que a pessoa eleve os braços e a resposta inadequada é a debilidade dos braços e por fim pedir para que fale uma frase onde a resposta inadequada é a incapacidade de falar ou a fala ilegível. Por fim foi explicado que o AVE é uma emergência médica e como tal, a qualquer suspeita deve-se acionar o SAMU (Serviço Móvel de Urgência) por meio do telefone 192, foi explicado que a ligação é gratuita e o serviço funciona 24 horas. Ao final do encontro foi feita avaliação de todos os assuntos e pedido que os idosos falassem de que modo às palestras contribuiu para seu aprendizado sobre os distúrbios neurológicos mais incidentes na terceira idade, onde foi um momento de discursão para verificar os pontos positivos e negativos da aplicação do plano, bem como, retirar dúvidas existentes sobre as temáticas. 


\section{CONCLUSÃO}

Conclui-se que as temáticas abordadas são de extrema importância para população, em especial a população idosa que tem maior incidência de acometimento por esses distúrbios sendo os índices de acometimento relativamente alto, e os conhecimentos acerca das temáticas, pouco difundidos. No grupo "Balança, mas não cai", foi possível observar que os participantes tinham um conhecimento vago sobre os temas propostos, sendo este fator preocupante por se tratarem de idosos, o grupo onde o acometimento é elevado, sendo essencial o conhecimento para contribuir para prevenção e tratamento das patologias, assim como, possíveis sequelas.

Os objetivos do plano de estágio foram alcançados, visto que foi possível perceber que após serem ministradas as palestras, a maioria dos participantes do grupo quando questionados souberam responder questões sobre os temas abordados, dando a certeza que as palestras foram de suma importância para formação de cidadãos multiplicadores das informações e dos conhecimentos que fora aprendido durante a aplicação do plano. Ao avaliar as temáticas desenvolvidas nos encontros pude perceber que os idosos são capazes de falar sobre a Doença de Alzheimer, Parkinson e AVE com enfoque na prevenção e nas manifestações clínicas e assim são capazes de buscar tratamento especializado quando necessário.

Diante do exposto, é importante que após a aplicação do plano de estágio, as temáticas continuem a ser disseminadas considerando sua importância no atual e futuro cenário brasileiro, mediante o envelhecimento populacional em contrapartida das taxas de mortalidade e natalidade, a fim de contribuir para promoção da saúde da população de modo geral, e em especial a terceira idade que é mais acometida por esses distúrbios. 


\section{REFERÊNCIAS BIBLIOGRÁFICAS}

BRASIL. Ministério da Saúde. Alzheimer. o que é, causas, sintomas, tratamento, diagnóstico e prevenção. Disponível em: https://saude.gov.br/saude-de-a-z/alzheimer. Acesso em: 10 de fevereiro de 2020.

BRASIL. Ministério da Saúde. Doenças crônicas não transmissíveis. Brasília: Ministério da Saúde, $2011 . \quad$ Disponível em: http://portalsaude.saude.gov.br/index.php/oministerio/principal/secretarias/svs/ doencascronicas-nao-transmissiveis. Acesso em: 25 de novembro de 2019.

BRASIL. Ministério da Saúde. Envelhecimento e saúde da pessoa idosa. Brasília: Ministério da Saúde, 2006 (Cadernos de Atenção Básica. n. 19).

BRASIL. Ministério da saúde. Plano de Ações Estratégicas para o Enfrentamento das Doenças Crônicas Não Transmissíveis (DCNT) no Brasil 2011-2022. Brasília: Ministério da Saúde, 2011.

BRASIL. Portaria Conjunta No 10, de 31 de outubro de 2017. Aprova o Protocolo Clínico e Diretrizes Terapêuticas da Doença de Parkinson. Diário Oficial da União, Brasília, DF, 08 de dez. de $2017 . \quad$ Disponível em: http://portalarquivos2.saude.gov.br/images/pdf/2017/novembro/14/Portaria-Conjunta- $\quad$ PCDTDoenca-de-Parkinson.pdf. Acesso em: 24 de novembro de 2019.

BRASIL. Portaria Conjunta No 13, DE 28 DE novembro de 2017. Aprova o Protocolo Clínico e Diretrizes Terapêuticas da Doença de Alzheimer. Diário Oficial da União,

Brasília, DF, 08 de dez. de 2017. Disponível em: http://www.in.gov.br/materia/lasset_publisher/Kujrw0TZC2Mb/content/id/867171/do1-2017-12-08-portaria-conjunta-n-13-de28-de-novembro-de-2017-867167. Acesso em: 25 de novembro de 2019.

CAETANO, L. A. O. et al. Alzheimer, sintomas e grupos: uma revisão integrativa. Rev. do NES. v. 14,n. 2, 2017.

CARVALHO, I. A.; DEODATO, L. F. F. Fatores de risco do Acidente vascular encefálico. Rev. Cient. FASETE, 2016.

HELLWIG, N. et al.Sintomas depressivos em idosos: estudo transversal de base populacional. Ciên. saú. cole. v. 21, n. 11, 2016.

NÓBREGA, I. R. A. P. et al. Fatores associados a depressão em idosos institucionalizados: revisão integrativa. Saú. Deb. v. 39, n. 105, p. 536-50, 2015.

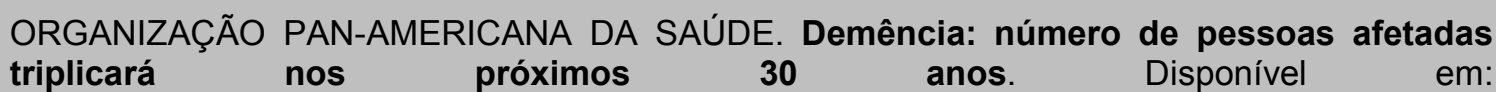
https://www.paho.org/bra/index.php?option=com_content\&view=article\&id=5560:dem encianumero-de-pessoas-afetadas-triplicara-nos-proximos-30-anos\&ltemid=839. Acesso em 24 de novembro de 2019.

ORGANIZAÇÃO PAN-AMERICANA DA SAÚDE. OPAS/OMS apresenta ações custo-efetivas para prevenir doenças crônicas, em encontro internacional. Disponível em: https://www.paho.org/bra/index.php?option=com_content\&view=article\&id=5730:opas-omsapresenta-acoes-custo-efetivas-para-prevenir-doencas-cronicas-em-encontro- 
internacional\&ltemid=839. Acesso em: 15 de janeiro 2020.

SALCI, M. A. et al. Educação em saúde para prevenção das complicações crônicas do diabetes mellitus na atenção primária. Esc Anna Nery. 2018.

SANTANA, M. T. M.; CHUN, R. Y. S. Linguagem e funcionalidade de adultos pós Acidente Vascular Encefálico (AVE): avaliação baseada na Classificação Internacional de Funcionalidade, Incapacidade e Saúde (CIF). CoDAS, v. 29, n. 1, 2017. 